Hospital Universitario Salamanca, Centro de Investigación del Cáncer, Instituto de Biología Molecular y Celular del Cáncer (Universidad de Salamanca-Consejo Superior de Investigaciones Científicas), Salamanca, Spain; Dana-Farber Cancer Institute, Boston; Millennium Pharmaceuticals, Cambridge, MA; Praxisklinik Dr Schlag, Würzburg; University of Münster, Münster, Germany; St Petersburg Botkin Moscow City Clinical Hospital, Moscow; Nizhnii Novgorod Region Clinical Hospital, Nizhnii Novgorod; St Petersburg Clinical Research Institute of Hematology \& Transfusiology, St Petersburg, Russian Federation; University of Athens School of Medicine, Athens, Greece; Rabin Medica Center, Petah-Tiqva, Israel; University Hospital, Prague, Czech Republic; University La Sapienza, Rome; Universita di Torino, Torino, Italy; Medical University of Lublin, Lublin, Poland; Myeloma Study Group, Belgian Hematological Society, Brussels; Johnson \& Johnson Pharmaceu tical Research \& Development, Beerse Belgium; People's Hospital, Peking University, Beijing, China; Johnson \& Johnson Pharmaceutical Research \& Development, Raritan, NJ; and Johnson \& Johnson Pharmaceutical Research \& Development, High Wycombe, United Kingdom.

Submitted September 8, 2009; accepted January 6, 2010; published online ahead of print at www.jco.org on April 5, 2010.

Supported by Johnson \& Johnson Pharmaceutical Research \& Development and Millennium Pharmaceuticals.

Presented in part in oral format at the 49th Annual Meeting of the American

Society of Hematology (ASH), December 8-11, 2007, Atlanta, GA; the 13th

Congress of the European Hematology Association, June 12-15, 2008, Copenhagen, Denmark; and the 50th Annual Meeting of the ASH, December 6-9, 2008, San Francisco, CA.

Written on behalf of the VISTA-MMY 3002 trial investigators.

Authors' disclosures of potential conflicts of interest and author contributions are found at the end of this article.

Corresponding author: Jesús F. San Miguel, MD, PhD, Hospital Universitario de Salamanca, Paseo San Vicente 58-182, 37007 Salamanca, Spain; e-mail: sanmigiz@usal.es.

(C) 2010 by American Society of Clinical Oncology

0732-183X/10/2813-2259/\$20.00

DOI: $10.1200 / \mathrm{JCO} .2009 .26 .0638$

\title{
Bortezomib Plus Melphalan and Prednisone Compared With Melphalan and Prednisone in Previously Untreated Multiple Myeloma: Updated Follow-Up and Impact of Subsequent Therapy in the Phase III VISTA Trial
}

Maria-Victoria Mateos, Paul G. Richardson, Rudolf Schlag, Nuriet K. Khuageva, Meletios A. Dimopoulos, Ofer Shpilberg, Martin Kropff, Ivan Spicka, Maria T. Petrucci, Antonio Palumbo, Olga S. Samoilova, Anna Dmoszynska, Kudrat M. Abdulkadyrov, Rik Schots, Bin Jiang, Dixie L. Esseltine, Kevin Liu, Andrew Cakana, Helgi van de Velde, and Jesús F. San Miguel

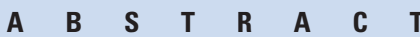

\section{Purpose}

The purpose of this study was to confirm overall survival (OS) and other clinical benefits with bortezomib, melphalan, and prednisone (VMP) versus melphalan and prednisone (MP) in the phase III VISTA (Velcade as Initial Standard Therapy in Multiple Myeloma) trial after prolonged follow-up, and evaluate the impact of subsequent therapies.

\section{Patients and Methods}

Previously untreated symptomatic patients with myeloma ineligible for high-dose therapy received up to nine 6-week cycles of VMP $(n=344)$ or MP $(n=338)$.

\section{Results}

With a median follow-up of 36.7 months, there was a $35 \%$ reduced risk of death with VMP versus MP (hazard ratio, 0.653; $P<.001$ ); median OS was not reached with VMP versus 43 months with MP; 3-year OS rates were $68.5 \%$ versus $54.0 \%$. Response rates to subsequent thalidomide$(41 \% \vee 53 \%)$ and lenalidomide-based therapies (59\% $\vee 52 \%)$ appeared similar after VMP or MP; response rates to subsequent bortezomib-based therapy were $47 \%$ versus $59 \%$. Among patients treated with VMP $(n=178)$ and MP $(n=233)$, median survival from start of subsequent therapy was 30.2 and 21.9 months, respectively, and there was no difference in survival from salvage among patients who received subsequent bortezomib, thalidomide, or lenalidomide. Rates of adverse events were higher with VMP versus MP during cycles 1 to 4 , but similar during cycles 5 to 9 . With VMP, 79\% of peripheral neuropathy events improved within a median of 1.9 months; $60 \%$ completely resolved within a median of 5.7 months.

\section{Conclusion}

VMP significantly prolongs OS versus MP after lengthy follow-up and extensive subsequent antimyeloma therapy. First-line bortezomib use does not induce more resistant relapse. VMP used upfront appears more beneficial than first treating with conventional agents and saving bortezomib- and other novel agent-based treatment until relapse.

\section{J Clin Oncol 28:2259-2266. (c) 2010 by American Society of Clinical Oncology}

\section{INTRODUCTION}

In the absence of curative therapy, the goal of treatment for multiple myeloma (MM) is prolonged overall survival (OS). Recent studies have suggested that over the past decade, the introduction of high-dose therapy for eligible patients age $\leq 65$ years and the novel agents bortezomib, thalidomide, and lenalidomide as rescue therapies has increased OS for patients with MM. ${ }^{1-3}$ However, improvements have been less pronounced in elderly patients ${ }^{1-3}$; the unmet need in this population may be addressed through optimal use of chemotherapy plus novel agents. ${ }^{1,4-7}$

Use of novel agent-based regimens in previously untreated patients ineligible for high-dose therapy has resulted in improved response rates and long-term outcomes, such as time to progression (TTP) and progression-free survival (PFS), compared with conventional regimens. ${ }^{8}$ However, analysis of OS is becoming increasingly complicated due to the impact of novel agents as rescue therapies. Indeed, only two ${ }^{4,9}$ of five $e^{4,6,9-11}$ phase III trials of thalidomide plus melphalan and prednisone (MPT) 
versus melphalan and prednisone (MP) have shown an OS benefit with MPT. Moreover, it has been suggested that first-line use of novel agents, although initially more effective than conventional chemotherapy regimens, could induce more resistant relapses. ${ }^{7,12-14}$ Accordingly, it is of increasing interest to analyze response to subsequent therapies in patients treated upfront with novel agents.

The first report of the international, randomized phase III VISTA trial demonstrated superior efficacy with bortezomib plus melphalan and prednisone (VMP) versus MP in previously untreated patients with MM ineligible for high-dose therapy. ${ }^{15}$ Follow-up was limited at first analysis (median, 16.3 months); 12\% of patients remained on therapy. ${ }^{15}$ We now present updated analyses of OS, time to next therapy (TNT), and treatment-free interval (TFI) to determine whether initial findings of superior efficacy with VMP versus MP are confirmed after prolonged follow-up and more extensive use of subsequent therapy. We also focus on survival from the start of subsequent therapy and response to rescue therapies, to explore whether VMP induced more resistant relapses than MP, and whether VMP used upfront appeared more beneficial than using conventional treatment upfront and saving novel agents until relapse.

\section{PATIENTS AND METHODS}

\section{Patients and Study Design}

VISTA study details have been reported. ${ }^{15}$ Briefly, patients with previously untreated $\mathrm{MM}$ who were ineligible for high-dose therapy were randomly assigned to receive nine 6-week cycles of VMP, comprising bortezomib $1.3 \mathrm{mg} / \mathrm{m}^{2}$ (days $1,4,8,11,22,25,29,32$, cycles 1 to 4 ; days 1 , $8,22,29$, cycles 5 to 9) plus melphalan $9 \mathrm{mg} / \mathrm{m}^{2}$ and prednisone $60 \mathrm{mg} / \mathrm{m}^{2}$ (days 1 to 4, all cycles), or MP. Patients with serum creatinine higher than $2 \mathrm{mg} / \mathrm{dL}$ or grade $\geq 2$ peripheral neuropathy or neuropathic pain by National Cancer Institute Common Toxicity Criteria for Adverse Events version 3.0 were excluded.

Response and progression were assessed every 3 weeks during treatment and then every 8 weeks until disease progression according to European Group for Blood and Marrow Transplantation criteria, ${ }^{16}$ using a central laboratory for M-protein quantification and immunofixation and a validated algorithm. Patients were then followed at least every 12 weeks for survival and subsequent therapy use. Safety was assessed until 30 days postlast dose of study drug; adverse events (AEs) were graded according to National Cancer Institute Common Toxicity Criteria for Adverse Events version 3.0. All patients provided written informed consent. Review boards at all participating institutions approved the study, which was conducted according to the provisions of the Declaration of Helsinki, the International Conference on Harmonization, and the Guidelines for Good Clinical Practice.

\section{Initial and Updated Analyses}

VISTA was stopped at the third preplanned interim analysis based on independent data monitoring committee recommendation, as the statistical boundary for the primary end point, TTP, had been crossed. Data cutoff was June 15, 2007; median follow-up was 16.3 months. ${ }^{15}$ Eighty patients remained on treatment (47 VMP, $33 \mathrm{MP}) .{ }^{15}$

Updated analyses were performed using data collected through March 16, 2009. After the study was stopped, formal centralized collection of $\mathrm{M}$-protein data was stopped and thus subsequent data on response rates, TTP,

\begin{tabular}{|c|c|c|c|c|}
\hline & \multicolumn{2}{|c|}{$\begin{array}{l}\text { Assessed for eligibility } \\
\qquad(N=755)\end{array}$} & \multirow{2}{*}{$\begin{array}{l}\text { Excluded } \\
(n=73)\end{array}$} & \\
\hline & \multicolumn{2}{|c|}{$\begin{array}{l}\text { Randomly assigned } \\
\qquad(n=682)\end{array}$} & & \\
\hline $\begin{array}{l}\text { Allocated to bortezomib plus } \\
\text { melphalan-prednisone } \\
\text { Received treatment } \\
\text { Did not receive treatment } \\
\text { Patient choice } \\
\text { Other }\end{array}$ & $\begin{array}{r}(n=344) \\
(n=340) \\
(n=4) \\
(n=3) \\
(n=1)\end{array}$ & \multicolumn{2}{|c|}{$\begin{array}{l}\text { Allocated to melphalan-prednisone } \\
\text { Received treatment } \\
\text { Did not receive treatment } \\
\text { Adverse event (renal insufficiency) }\end{array}$} & $\begin{array}{r}(n=338) \\
(n=337) \\
(n=1)\end{array}$ \\
\hline $\begin{array}{l}\text { Treatment completed } \\
\text { Discontinued treatment } \\
\text { Progressive disease } \\
\text { Adverse event } \\
\text { Patient choice } \\
\text { Death } \\
\text { Maintenance of complete response } \\
\text { Other }\end{array}$ & $\begin{array}{r}(n=199) \\
(n=141) \\
(n=24) \\
(n=52) \\
(n=32) \\
(n=14) \\
(n=9) \\
(n=10)\end{array}$ & $\begin{array}{l}\text { Treatmen } \\
\text { Discontin } \\
\text { Progress } \\
\text { Adverse } \\
\text { Patient c } \\
\text { Death } \\
\text { Mainten } \\
\text { Other }\end{array}$ & $\begin{array}{l}\text { leted } \\
\text { eatment } \\
\text { sease } \\
\text { f complete response }\end{array}$ & $\begin{array}{r}(n=169) \\
(n=168) \\
(n=73) \\
(n=48) \\
(n=19) \\
(n=17) \\
(n=1) \\
(n=10)\end{array}$ \\
\hline $\begin{array}{l}\text { Analyzed for response } \\
\text { Excluded from response analysis } \\
\text { No measurable disease at baseline }\end{array}$ & $\begin{array}{r}(n=337) \\
(n=3) \\
(n=3)\end{array}$ & $\begin{array}{l}\text { Analyzed } \\
\text { Excluded } \\
\text { No measu }\end{array}$ & $\begin{array}{l}\text { ponse } \\
\text { esponse analysis } \\
\text { disease at baseline }\end{array}$ & $\begin{array}{r}(n=331) \\
(n=6) \\
(n=6)\end{array}$ \\
\hline $\begin{array}{l}\text { Received subsequent therapy } \\
\text { Did not receive subsequent therapy } \\
\text { Not yet required } \\
\text { Died due to PD prior to receiving } \\
\text { subsequent therapy }\end{array}$ & $\begin{array}{l}(n=178) \\
(n=166) \\
(n=159) \\
(n=7)\end{array}$ & $\begin{array}{l}\text { Received } \\
\text { Did not re } \\
\text { Not yet } r \\
\text { Died due } \\
\text { subseq }\end{array}$ & $\begin{array}{l}\text { quent therapy } \\
\text { subsequent therapy } \\
\text { d } \\
\text { prior to receiving } \\
\text { erapy }\end{array}$ & $\begin{array}{r}(\mathrm{n}=233) \\
(\mathrm{n}=105) \\
(\mathrm{n}=92) \\
(\mathrm{n}=13)\end{array}$ \\
\hline
\end{tabular}

Fig 1. CONSORT diagram. PD, progressive disease. 
and PFS were not updated because the information would not have been based on the same stringent methodology as the initial analysis. Data collection continued for OS, subsequent therapy use, best responses to subsequent therapies as reported by investigators, and safety, including improvement/resolution of AEs (until 30 days post-treatment completion).

\section{Statistical Analysis}

OS (random assignment until death), TNT (random assignment until first dose of subsequent anti-MM therapy), and TFI (last dose of VISTA study drug until first dose of subsequent anti-MM therapy) were analyzed using Kaplan-Meier methodology. Time-to-event data were compared between arms using stratified log-rank tests in intent-to-treat analyses. In posthoc analyses among patients treated with VMP, OS was evaluated within subgroups with/without poor prognostic characteristics, including advanced age, impaired renal function, and high-risk cytogenetics. In exploratory posthoc analyses, survival was evaluated from random assignment and from start of subsequent therapy by arm in patients who had received any subsequent therapy and according to subsequent therapy received. Safety was analyzed in all patients who received at least one dose of study drug. Rates of treatment-emergent AEs were analyzed overall and by treatment cycle.

\section{RESULTS}

\section{Patient Characteristics and Disposition}

A total of 682 patients were randomly assigned to receive VMP $(\mathrm{n}=344)$ or MP $(\mathrm{n}=338)$. Baseline demographics and disease characteristics were previously reported, ${ }^{15}$ and are summarized in Appendix Table A1 (online only). Overall, median age was 71 years, $30 \%$ of patients were age $\geq 75$ years, $34 \%$ had International Staging System ${ }^{17}$ stage III MM, and 33\% had $\beta_{2}$-microglobulin higher than $5.5 \mathrm{mg} / \mathrm{L}$. At the initial analysis, VMP was superior to MP across all efficacy end points, including response rate $(71 \% v 35 \%)$, complete response (CR) rate $(30 \% v 4 \%)$, TTP (median, $24.0 v 16.6$ months; hazard ratio [HR], $0.48 ; P<.001)$, and OS (HR, 0.61; $P=.008) .{ }^{15}$ Data not updated (see Patients and Methods) are summarized in Appendix Table A2 (online only).

Updated patient disposition at data cutoff for this analysis (March 16, 2009) is shown in Figure 1 (CONSORT). All patients have completed VISTA study treatment. Median number of treatment

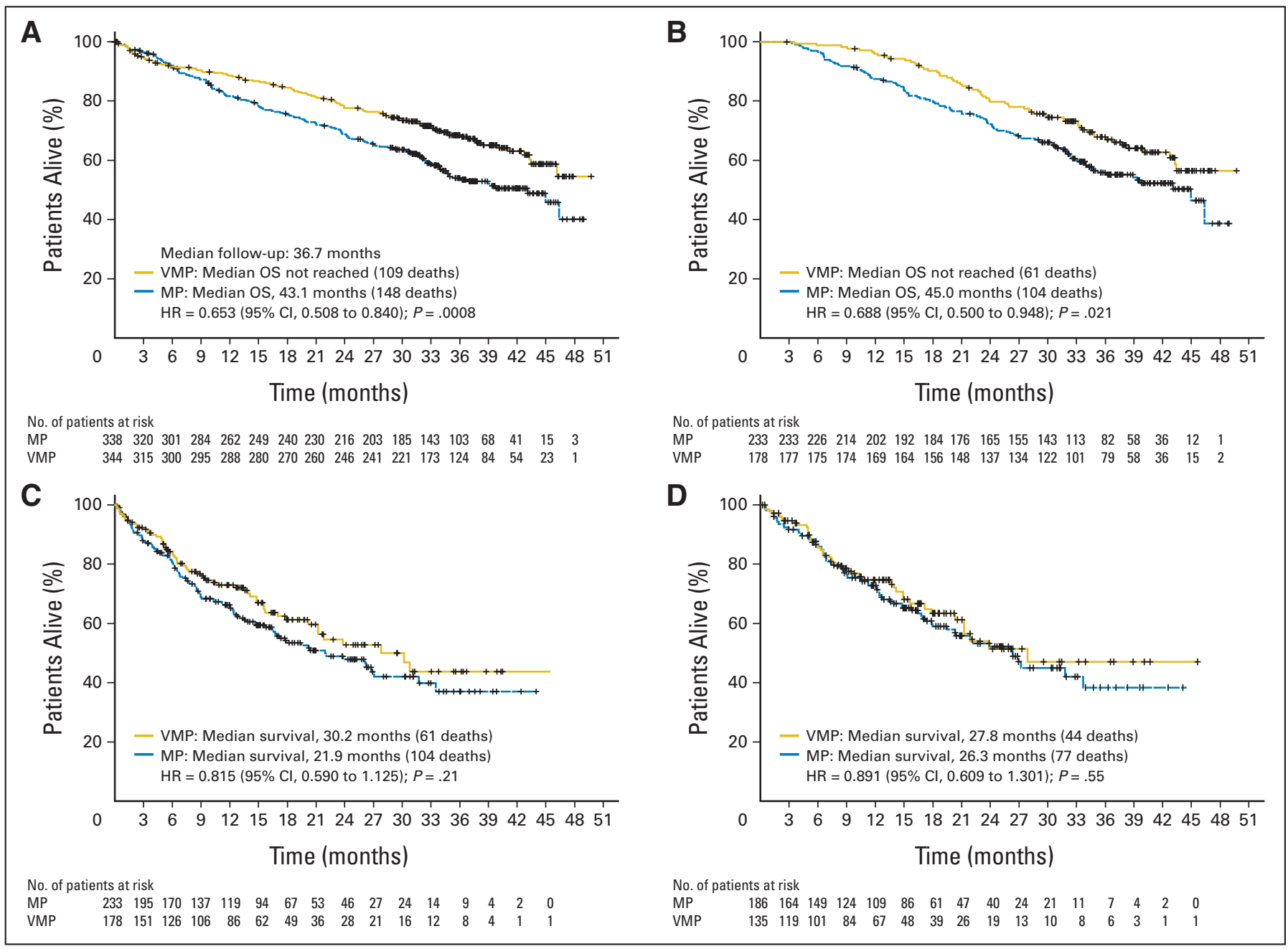

Fig 2. Overall survival (OS) from random assignment in the bortezomib, melphalan, and prednisone (VMP) and melphalan and prednisone (MP) arms in (A) the overall population (ITT analysis), and (B) only patients randomly assigned to VMP or MP who had received subsequent therapy by data cutoff for the present analysis. Survival from the start of second-line therapy in (C) all patients who had received subsequent therapy, and (D) patients who received any subsequent novel agents. HR, hazard ratio. 


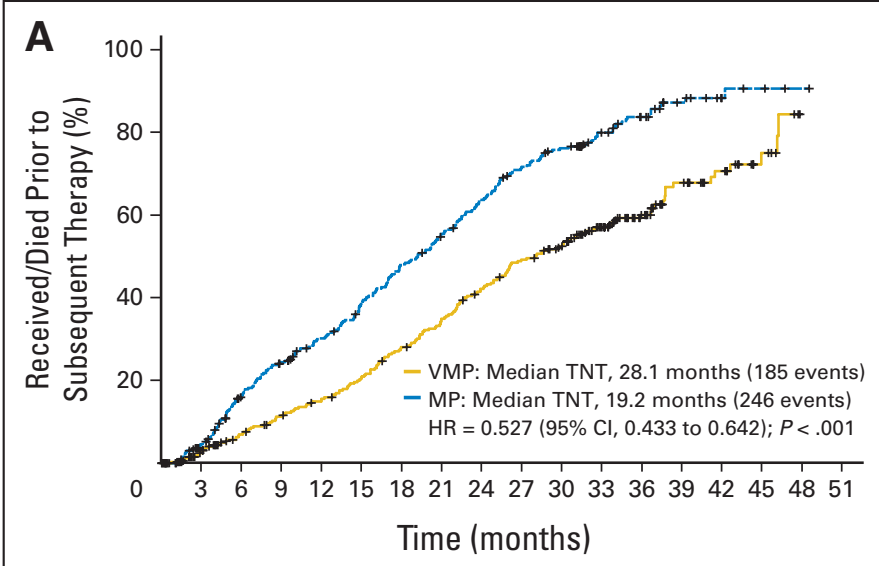

No. of patients at risk

$\begin{array}{llllllllllllllllll}\text { MP } & 338 & 307 & 259 & 229 & 204 & 177 & 149 & 126 & 101 & 77 & 59 & 40 & 27 & 12 & 6 & 3 & 1\end{array}$ $\begin{array}{lrlllllllllllllllll}\text { VMP } & 344 & 310 & 283 & 267 & 252 & 235 & 211 & 189 & 165 & 143 & 126 & 88 & 56 & 30 & 21 & 9 & 0\end{array}$

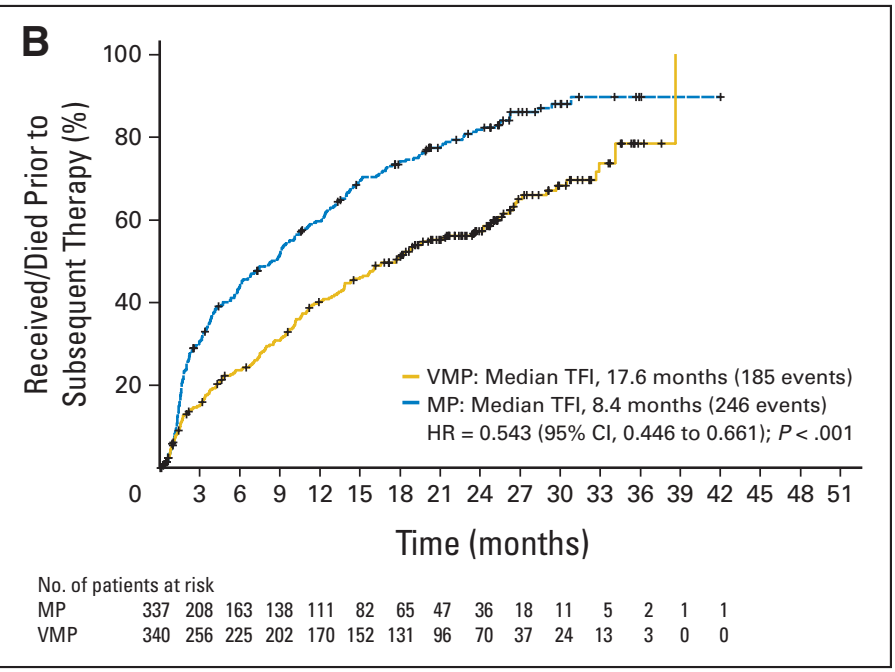

Fig 3. (A) Time to next therapy (TNT) and (B) treatment-free interval (TFI) with bortezomib, melphalan, and prednisone (VMP) and melphalan and prednisone (MP). HR, hazard ratio.

cycles administered was nine (50 weeks) and eight (48 weeks) in the VMP and MP arms, respectively.

\section{os}

After median follow-up of 36.7 months, OS was prolonged with VMP versus MP (HR, 0.653; $P<.001)$, with VMP associated with a $35 \%$ reduced risk of death. Median OS was not estimable versus 43.1 months in the VMP and MP arms, respectively, with 109 (32\%) and 148 (44\%) patients having died (Fig 2A); 3-year OS rates were 68.5\% versus $54.0 \%$, respectively. The improved OS with VMP versus MP in patient subgroups predefined by age, sex, race, $\beta_{2}$-microglobulin, albumin, region, International Staging System stage, and creatinine clearance is shown in Appendix Figure A1 (online only).

\section{Subsequent Therapy}

At data cutoff, 178 (52\%) and 233 patients (69\%) in the VMP and MP arms, respectively, had received subsequent therapy. TNT and TFI with VMP versus MP are shown in Figure 3; median TNT was 28.1 months versus 19.2 months (HR, 0.527; $P<.001)$, and median TFI was 17.6 months versus 8.4 months $(\mathrm{HR}, 0.543 ; P<.001)$. In the VMP and MP arms, $42.7 \%$ and $18.1 \%$ of patients, respectively, had a TFI of $\geq 2$ years.

Novel agents received as part of subsequent anti-MM therapy are summarized in Appendix Table A3 (online only). Among patients who received subsequent therapy in the VMP and MP arms, respectively, 43 (24\%) and $116(50 \%)$ received bortezomib, 81 (46\%) and $110(47 \%)$ received thalidomide, and $57(32 \%)$ and $30(13 \%)$ received

\begin{tabular}{|c|c|c|c|c|c|c|c|c|c|c|c|c|}
\hline \multirow{4}{*}{$\begin{array}{l}\text { Subsequent Therapy and } \\
\text { Responses Achieved }\end{array}$} & \multicolumn{12}{|c|}{ Arm by Line } \\
\hline & \multicolumn{6}{|c|}{ VMP } & \multicolumn{6}{|c|}{$\mathrm{MP}$} \\
\hline & \multicolumn{2}{|c|}{ 2nd } & \multicolumn{2}{|c|}{$\geq 3 r d$} & \multicolumn{2}{|c|}{ Any* } & \multicolumn{2}{|c|}{ 2nd } & \multicolumn{2}{|c|}{$\geq 3 r d$} & \multicolumn{2}{|c|}{ Any* } \\
\hline & No. & $\%$ & No. & $\%$ & No. & $\%$ & No. & $\%$ & No. & $\%$ & No. & $\%$ \\
\hline Bortezomib-based therapy (No. with data/No. total) & $22 / 25$ & & $17 / 21$ & & $36 / 43$ & & $76 / 82$ & & $49 / 50$ & & $109 / 116$ & \\
\hline $\mathrm{CR}+\mathrm{PR}+$ & 9 & 41 & 8 & 47 & 17 & 47 & 45 & 59 & 27 & 55 & 64 & 59 \\
\hline $\mathrm{CR}+$ & 2 & 9 & 0 & & 2 & 6 & 6 & 8 & 5 & 10 & 9 & 8 \\
\hline Thalidomide-based therapy (No. with data/No. total) & $63 / 71$ & & $19 / 23$ & & $73 / 81$ & & $73 / 84$ & & $38 / 44$ & & $97 / 110$ & \\
\hline $\mathrm{CR}+\mathrm{PR}+$ & 23 & 37 & 10 & 53 & 30 & 41 & 34 & 47 & 21 & 55 & 51 & 53 \\
\hline $\mathrm{CR}+$ & 2 & 3 & 0 & & 2 & 3 & 1 & 1 & 4 & 11 & 5 & 5 \\
\hline Lenalidomide-based therapy (No. with data/No. total) & $22 / 26$ & & $27 / 34$ & & $46 / 57$ & & $9 / 10$ & & $14 / 21$ & & $23 / 30$ & \\
\hline $\mathrm{CR}+\mathrm{PR} \dagger$ & 16 & 73 & 12 & 44 & 27 & 59 & 6 & 67 & 6 & 43 & 12 & 52 \\
\hline $\mathrm{CR}+$ & 2 & 9 & 2 & 7 & 4 & 9 & 1 & 11 & 0 & & 1 & 4 \\
\hline \multicolumn{13}{|c|}{$\begin{array}{l}\text { Abbreviations: VMP, bortezomib, melphalan, and prednisone; MP, melphalan and prednisone; CR, complete response; PR, partial response. } \\
\text { *Patients could have received more than one agent, either within the same combination regimen, as part of consecutive separate regimens within the same line } \\
\text { of therapy, or as part of separate regimens within different subsequent lines of therapy. These patients are included in each of the relevant groups. } \\
\text { tPercentage response rates calculated using the number of patients with data as the denominator. No. with data represents the numbers of patients for whom } \\
\text { the investigators reported the response to subsequent therapy; in each arm, responses to subsequent therapy were unknown or missing for some of the total } \\
\text { number of patients who received the subsequent therapy. }\end{array}$} \\
\hline
\end{tabular}


lenalidomide. Investigator-reported best responses to novel agentbased subsequent therapies are summarized in Table 1. Response rates to second-line bortezomib-, thalidomide-, and lenalidomide-based therapies were $41 \%, 37 \%$, and $73 \%$, respectively, after VMP, and 59\%, $47 \%$, and $67 \%$, respectively, after MP; respective response rates to therapies received at third line and beyond were 47\%, 53\%, and 44\% after VMP, and 55\%, 55\%, and 43\% after MP. Efficacy of bortezomibbased re-treatment as second-line therapy in patients randomly assigned to VMP was evaluated according to TFI. Among 16 patients with TFI $\leq 12$ months, response rate was $25 \%$, including $6 \% \mathrm{CR}$. Among nine patients with TFI longer than 12 months, response rate (in seven with response reported) was 71\%, including 14\% CR.

Figure $2 \mathrm{~B}$ shows $\mathrm{OS}$ from initial random assignment among patients treated with VMP and MP who received subsequent therapy (HR, $0.688 ; P=.021$ ); 3-year OS rates were $67.9 \%$ and $55.9 \%$, respectively. The OS advantage with VMP was also seen versus the subgroup of patients treated with MP who received bortezomib as second-line therapy (data not shown). Median survival from start of subsequent therapy was 30.2 and 21.9 months after VMP and MP, respectively (Fig 2C; HR, 0.815; $P=.21$ ). Notably, survival from start of subse- quent therapy was similar after VMP versus MP among patients who received any novel agents as subsequent therapy (Fig 2D; median $27.8 v 26.3$ months; HR, 0.891; $P=.55$ ) and among patients who received subsequent thalidomide and lenalidomide but no bortezomib (median 27.8 months $v$ not reached; HR, 0.766; $P=.35$ ).

\section{VMP Subgroup Analyses}

Within the VMP arm, OS was longer among patients age younger than 75 versus $\geq 75$ years $(\mathrm{HR}, 1.664 ; P=.011$; 3 -year OS: $74.1 \%$ 55.5\%; Fig 4A). By contrast, there were no statistically significant differences although there were trends to longer OS among patients with creatinine clearance $\geq 60$ versus fewer than $60 \mathrm{~mL} / \mathrm{min}$ (HR, 1.291; $P=.238$; 3-year OS: 74.5\% v 63.1\%; Fig 4B), and patients with standard risk versus high-risk cytogenetics ( $\mathrm{HR}, 1.346 ; P=.399$; 3-year OS 71.6\% v 56.1\%; Fig 4C).

Novel agents received as part of subsequent therapy according to age, baseline creatinine clearance, and cytogenetics are summarized in Appendix Table A4 (online only). Survival from start of subsequent therapy appeared shorter among patients with creatinine

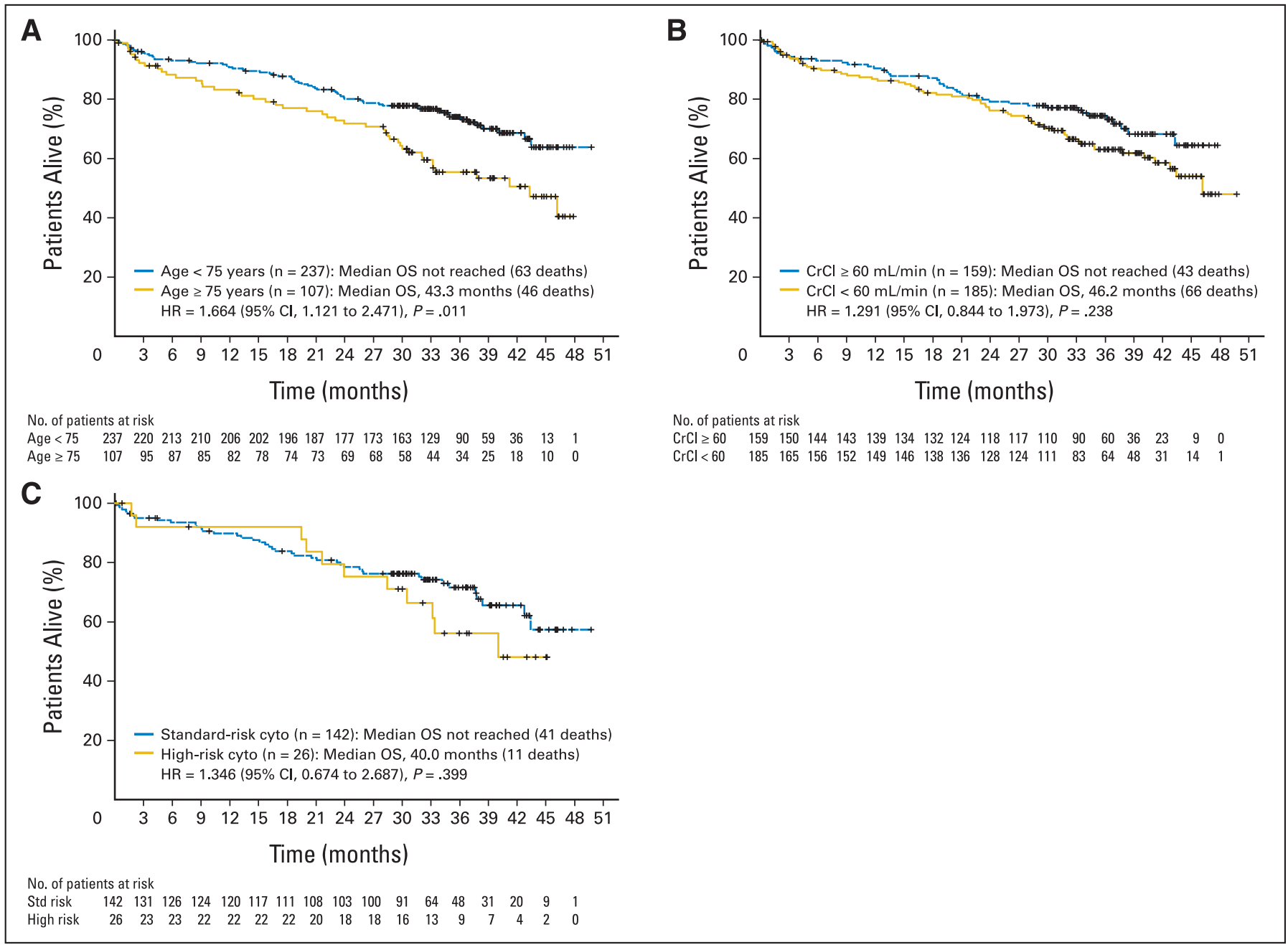

Fig 4. Overall survival (OS) from random assignment in the bortezomib, melphalan, and prednisone arm in patients (A) age $\leq 75$ and older than 75 years, (B) with creatinine clearance $(\mathrm{CrCl}) \geq 60$ and shorter than $60 \mathrm{~mL} / \mathrm{min},(\mathrm{C})$ with standard-risk (std) and high-risk (t(4;14), t(14;16), del(17p)) cytogenetics (cyto) by fluorescence in situ hybridization. HR, hazard ratio. 


\begin{tabular}{|c|c|c|c|c|}
\hline \multirow[b]{3}{*}{ Adverse Event } & \multicolumn{4}{|c|}{ Arm by Grade (\%) } \\
\hline & \multicolumn{2}{|c|}{$\begin{array}{c}\text { VMP } \\
(n=340)\end{array}$} & \multicolumn{2}{|c|}{$\begin{array}{c}\text { MP } \\
(n=337)\end{array}$} \\
\hline & 3 & 4 & 3 & 4 \\
\hline Neutropenia & 29 & 11 & 23 & 15 \\
\hline Thrombocytopenia & 20 & 18 & 16 & 15 \\
\hline Anemia & 16 & 3 & 20 & 8 \\
\hline Leukopenia & 21 & 3 & 16 & 4 \\
\hline Lymphopenia & 14 & 6 & 9 & 2 \\
\hline Peripheral sensory neuropathy & 13 & $<1$ & 0 & 0 \\
\hline Neuralgia & 8 & 1 & $<1$ & 0 \\
\hline Fatigue & 7 & 1 & 2 & 0 \\
\hline Diarrhea & 7 & 1 & 1 & 0 \\
\hline Pneumonia & 5 & 2 & 4 & 1 \\
\hline Hypokalemia & 6 & 1 & 2 & 1 \\
\hline Asthenia & 6 & $<1$ & 3 & 0 \\
\hline
\end{tabular}

Abbreviations: VMP, bortezomib, melphalan, and prednisone; MP, melphalan and prednisone.

clearance shorter than 60 versus $\geq 60 \mathrm{~mL} / \mathrm{min}$ who received subsequent thalidomide and lenalidomide but no bortezomib (Appendix Fig A2A, online only); no difference was apparent among the small number of patients who received subsequent bortezomib but no thalidomide and lenalidomide (Appendix Fig A2B). TNT was similar between patients with high-risk versus standard-risk cytogenetics (median $24.1 v 27.4$ months; HR, 1.478; $P=.17$ ); survival from start of subsequent therapy appeared similar between cytogenetic risk groups among patients who received any subsequent novel agent-based therapy (Appendix Fig A2C) but substantially shorter in two high-risk (6 and 15.5 months) versus 17 standard-risk patients (median, 30.8 months) who received no subsequent novel agents.

\section{Safety}

Safety profiles were similar to those reported at the initial analysis. ${ }^{15}$ Rates of on-study deaths were similar between the VMP ( $\mathrm{n}=19$; $6 \%)$ and MP $(\mathrm{n}=14 ; 4 \%)$ arms, including $2 \%$ treatment-related deaths on each arm. Rates of treatment discontinuation due to AEs remained similar (15\%/14\% in the VMP/MP arms). Grade $3 / 4 \mathrm{AEs}$ that occurred at an incidence of $\geq 5 \%$ in either arm are presented in Table 2 .

The rate of peripheral neuropathy with VMP was almost identical to that reported at the initial analysis. ${ }^{15}$ After prolonged follow-up, $79 \%$ of peripheral neuropathy events had improved/resolved within a median of 1.9 months, while $60 \%$ completely resolved within a median of 5.7 months. Among 159 patients who had peripheral neuropathy while receiving VMP, 21 (13\%), 36 (23\%), and 39 (25\%) have received subsequent bortezomib-, thalidomide-, and lenalidomidebased therapy, respectively, compared with 22 (12\%), 45 (24\%), and 18 (10\%), respectively, of the 185 patients who did not report peripheral neuropathy. Among 21 patients who had peripheral neuropathy during VMP therapy and subsequently received bortezomib-based therapy, 16 (76\%) had at least one event improved/resolved, including 12 (57\%) with at least one event resolved, by the start of subsequent therapy.

VMP and MP safety profiles by treatment cycle are summarized in Table 3. Rates of AEs were higher with VMP versus MP during cycles 1 to 4, but similar during cycles 5 to 9. Rates of AEs, grade $\geq 3$ AEs, serious AEs, and discontinuations due to AEs in the VMP arm
Table 3. Tolerability of VMP and MP Therapy by Treatment Cycle

\begin{tabular}{|c|c|c|c|c|c|c|}
\hline \multirow[b]{3}{*}{ Parameter } & \multicolumn{6}{|c|}{ Cycle (\%) } \\
\hline & \multicolumn{3}{|c|}{ VMP } & \multicolumn{3}{|c|}{ MP } \\
\hline & All & $1-4$ & $5-9$ & All & $1-4$ & $5-9$ \\
\hline No. of patients & 340 & 340 & 249 & 337 & 337 & 234 \\
\hline $\begin{array}{l}\text { Any treatment-emergent adverse } \\
\text { event }\end{array}$ & 99 & 99 & 91 & 97 & 95 & 88 \\
\hline Grade 1 maximum severity & 1 & NA & NA & 3 & NA & NA \\
\hline Grade 2 maximum severity & 8 & NA & NA & 14 & NA & NA \\
\hline $\begin{array}{l}\text { Any grade } \geq 3 \text { treatment-emergent } \\
\text { adverse event }\end{array}$ & 91 & 85 & 62 & 80 & 66 & 63 \\
\hline Grade 3 maximum severity & 53 & NA & NA & 44 & NA & NA \\
\hline Grade 4 maximum severity & 29 & NA & NA & 28 & NA & NA \\
\hline Any serious adverse event & 46 & 40 & 14 & 36 & 29 & 16 \\
\hline $\begin{array}{l}\text { Discontinuation of regimen } \\
\text { because of adverse events }\end{array}$ & $15^{*}$ & $12^{*}$ & $5^{*}$ & 14 & 8 & 7 \\
\hline $\begin{array}{l}\text { Abbreviations: VMP, bortezomib, m } \\
\text { and prednisone; NA, not assessable } \\
\text { "Data represent discontinuation of } \\
\text { patients discontinued bortezomib bu } \\
\text { cycles } 1-4 \text { and } 5-9 \text {, respectively. }\end{array}$ & ma & med & ac & . M & $5 \%$ & alan \\
\hline
\end{tabular}

were lower during cycles 5 to 9 compared with cycles 1 to 4 . In the MP arm, rates of grade $\geq 3$ AEs and discontinuations due to AEs were similar in cycles 1 to 4 and cycles 5 to 9 .

\section{DISCUSSION}

The results of this updated analysis of the international phase III VISTA trial after prolonged follow-up (median, 36.7 months) confirm that VMP results in significantly longer OS compared with MP in previously untreated patients with MM who are ineligible for highdose therapy. This OS benefit was seen consistently across patient subgroups predefined by baseline characteristics, including age, disease stage, and creatinine clearance.

This confirmed survival advantage represents an important finding, as an OS benefit has not been consistently reported in other studies of novel agent-based regimens versus MP. For example, an OS benefit was reported in two studies of MPT versus MP by the Intergroupe Francophone du Myélome, ${ }^{4,9}$ but not in three other similar phase III studies. ${ }^{6,10,11}$ One reason suggested for the lack of OS benefit with MPT was the confounding impact of subsequent therapies containing novel agents in patients receiving MP upfront. ${ }^{6,11}$

However, in VISTA, the OS benefit with VMP versus MP was seen both overall and in an analysis restricted to patients who had received subsequent therapy, despite $50 \%$ of patients treated with MP being rescued with bortezomib-based therapy in the relapsed setting. Indeed, this benefit was retained with VMP versus MP followed by bortezomib as second-line therapy. Thus, our results indicate that using bortezomib-based treatment in the first-line setting provides greater survival benefit to patients compared with the approach of administering first-line treatment with conventional agents and saving bortezomib- and other novel agent-based treatment for salvage.

Patients receiving VMP experienced greater clinical benefit versus those receiving MP in terms of TNT and TFI. Among patients who had received subsequent therapy, taking into consideration potential differences in the order in which subsequent therapies were received, as well as the possible confounding factor of the different proportions 
of patients on each arm who had received subsequent therapy, response rates to subsequent thalidomide- and lenalidomide-based therapies appeared similar between arms. Furthermore, re-treatment with bortezomib-based therapies after VMP was effective; the $47 \%$ response rate reflects similar findings in studies of bortezomib-based re-treatment in relapsed MM. ${ }^{18-22}$ As might be expected, the response rate to subsequent bortezomib-based therapies appeared somewhat higher in bortezomib-naïve patients after MP (59\%). Survival from start of subsequent therapy was not shorter, and may even be longer, among patients randomly assigned to VMP versus MP (median $30.2 v$ 21.9 months). Importantly, there was no difference between arms in survival from start of subsequent therapy among patients who received any subsequent novel agents. Our findings indicate that patients relapsing after bortezomib-based therapy are not intrinsically more resistant to subsequent therapies, and can be as successfully treated with subsequent immunomodulator-based therapies, compared with those relapsing after traditional chemotherapy with MP; VMP treatment does not appear to select a more resistant clone.

Within the VMP arm, OS was longer among patients younger than 75 versus $\geq 75$ years, reflecting the expected adverse impact on survival of advanced age. Nonstatistically significant differences in OS were seen among patients treated with VMP with or without renal impairment and high-risk cytogenetics. Notably, OS curves appeared similar until approximately 24 to 28 months (median TTP/TNT), and subsequently appeared to show greater divergence with a trend to shorter OS for patients with adverse characteristics. Regarding this apparent impact of renal impairment, our findings indicated that among patients treated with VMP who received subsequent therapy including thalidomide and lenalidomide but not bortezomib, survival from start of subsequent therapy was longer in patients with creatinine clearance $\geq 60$ versus lower than $60 \mathrm{~mL} / \mathrm{min}$. By contrast, survival did not appear affected by renal function among patients who received subsequent bortezomib but not thalidomide and lenalidomide. However, these are retrospective analyses, and should thus be interpreted with caution. Regarding outcomes among patients treated with VMP with high-risk cytogenetics, we observed similar survival from start of subsequent therapy among 15 high-risk and 57 standard-risk patients who received novel agent-based rescue therapies. Thus, a possible interpretation of the trend toward shorter OS among patients with high-risk versus standard-risk cytogenetics is that there was a trend toward earlier receipt of subsequent therapy in high-risk patients, and that two of 17 high-risk patients were not exposed to novel agents after relapse and had notably poor survival from start of subsequent therapy. However, the small number of patients treated with VMP with high-risk cytogenetics precludes drawing any firm conclusions. At the initial analysis, response rates and TTP with VMP were similar among patients with or without these adverse characteristics. ${ }^{15}$ Overall, our findings reflect those from a pilot phase I to II study of $\mathrm{VMP}^{23}$ and preliminary data from other phase III studies of modified VMP regimens, ${ }^{24,25}$ in which high-risk cytogenetics did not affect response rates, ${ }^{23-25} \mathrm{TTP}^{23}$ or PFS. ${ }^{25}$ Similarly, high-risk cytogenetics did not affect response rates with other bortezomib-based regimens in previously untreated patients with $\mathrm{MM}^{26-29}$

At this updated analysis, after completion of therapy among 80 patients who were ongoing at the initial analysis, the overall safety profiles of VMP and MP were essentially unchanged. VMP was generally well-tolerated, with manageable toxicities. Our analysis of treatment-emergent AEs by treatment cycle demonstrated that toxicities associated with VMP were substantially lower during cycles 5 to 9 versus cycles 1 to 4 and similar to those associated with MP. Thus, while initial substantial tumor-burden reduction with twice-weekly bortezomib during cycles 1 to 4 was associated with somewhat more frequent toxicities with VMP versus MP, tolerability was improved and comparable to MP during weekly bortezomib maintenance (cycles 5 to 9), with improved responses and an increased CR rate from the end of cycle $4 .{ }^{30}$ Regimens using weekly bortezomib dosing schedules are currently under investigation ${ }^{24,25}$ and showing a clear reduction in toxicity, but longer follow-up is needed to confirm if efficacy is maintained.

After prolonged follow-up, the rate of improvement or resolution of peripheral neuropathy events on the VMP arm was 79\%, demonstrating that this toxicity is reversible in the majority of cases. Indeed, $60 \%$ of events had resolved completely to baseline by data cutoff, within a median of 5.7 months. Our findings are supported by data on reversibility of peripheral neuropathy with single-agent bortezomib in first-line ${ }^{31}$ and relapsed ${ }^{32} \mathrm{MM}$.

In conclusion, this updated analysis of VISTA supports the strategy of using bortezomib-based treatment as first-line therapy instead of as salvage after upfront conventional therapy. Our results confirm the significant survival benefit and the other clinical benefits of TNT and TFI with VMP versus MP. The survival benefit was demonstrated even with the use of subsequent novel agent-based therapies in patients treated with MP alone upfront. Indeed, salvage therapies appeared similarly effective in patients initially randomly assigned to VMP or MP, demonstrating that the use of bortezomib upfront does not preclude the successful use of novel agents at relapse. Moreover, postrelapse survival even appeared longer after VMP, indicating that first-line bortezomib use does not induce more resistant relapse.

\section{AUTHORS' DISCLOSURES OF POTENTIAL CONFLICTS} OF INTEREST

Although all authors completed the disclosure declaration, the following author $(s)$ indicated a financial or other interest that is relevant to the subject matter under consideration in this article. Certain relationships marked with a " $U$ " are those for which no compensation was received; those relationships marked with a "C" were compensated. For a detailed description of the disclosure categories, or for more information about ASCO's conflict of interest policy, please refer to the Author Disclosure Declaration and the Disclosures of Potential Conflicts of Interest section in Information for Contributors.

Employment or Leadership Position: Dixie L. Esseltine, Millennium Pharmaceuticals (C); Kevin Liu, Johnson \& Johnson Pharmaceutical Research \& Development (C); Andrew Cakana, Johnson \& Johnson Pharmaceutical Research \& Development (C); Helgi van de Velde, Johnson \& Johnson Pharmaceutical Research \& Development (C) Consultant or Advisory Role: Paul G. Richardson, Celgene (C), Johnson \& Johnson (C), Millennium Pharmaceuticals (C), Bristol-Myers Squibb (C); Meletios A. Dimopoulos, Ortho Biotech (C); Martin Kropff, Janssen-Cilag (C), Celgene (C); Rik Schots, Celgene (C); Jesús F. San Miguel, Jansenn-Cilag (C), Celgene (C), Millennium Pharmaceuticals (C) Stock Ownership: Dixie L. Esseltine, Millennium Pharmaceuticals, Johnson \& Johnson; Kevin Liu, Johnson \& Johnson; Andrew Cakana, Johnson \& Johnson Pharmaceutical Research \& Development; Helgi van de Velde, Johnson \& Johnson Pharmaceutical Research \& Development Honoraria: Maria-Victoria Mateos, Janssen-Cilag, Celgene; Meletios A. Dimopoulos, Ortho Biotech, Millennium Pharmaceuticals; Martin Kropff, Janssen-Cilag, Celgene; Ivan Spicka, Janssen-Cilag; Maria T. Petrucci, Janssen-Cilag, Celgene; Antonio Palumbo, Celgene, Janssen-Cilag; Rik Schots, Celgene Research Funding: Paul G. Richardson, Millennium Pharmaceuticals; Anna Dmoszynska, Johnson \& Johnson Expert Testimony: None Other Remuneration: None 


\section{AUTHOR CONTRIBUTIONS}

Conception and design: Maria-Victoria Mateos, Paul G. Richardson, Meletios A. Dimopoulos, Dixie L. Esseltine, Helgi van de Velde,

Jesús F. San Miguel

Provision of study materials or patients: Maria-Victoria Mateos, Paul G. Richardson, Rudolf Schlag, Nuriet K. Khuageva, Meletios A. Dimopoulos, Ofer Shpilberg, Martin Kropff, Ivan Spicka, Maria T. Petrucci, Antonio Palumbo, Olga S. Samoilova, Anna Dmoszynska, Kudrat M. Abdulkadyrov, Rik Schots, Bin Jiang, Dixie L. Esseltine, Jesús F. San Miguel

Collection and assembly of data: Maria-Victoria Mateos, Paul G. Richardson, Rudolf Schlag, Nuriet K. Khuageva, Martin Kropff, Ivan
Spicka, Olga S. Samoilova, Kudrat M. Abdulkadyrov, Bin Jiang, Kevin Liu, Andrew Cakana, Helgi van de Velde, Jesús F. San Miguel Data analysis and interpretation: Maria-Victoria Mateos, Meletios A. Dimopoulos, Ofer Shpilberg, Dixie L. Esseltine, Kevin Liu, Andrew Cakana, Helgi van de Velde, Jesús F. San Miguel

Manuscript writing: Maria-Victoria Mateos, Paul G. Richardson, Meletios A. Dimopoulos, Ivan Spicka, Kevin Liu, Helgi van de Velde, Jesús F. San Miguel

Final approval of manuscript: Maria-Victoria Mateos, Paul G. Richardson, Rudolf Schlag, Nuriet K. Khuageva, Meletios A. Dimopoulos, Ofer Shpilberg, Martin Kropff, Ivan Spicka, Maria T. Petrucci, Antonio Palumbo, Olga S. Samoilova, Anna Dmoszynska, Kudrat M. Abdulkadyrov, Rik Schots, Bin Jiang, Dixie L. Esseltine, Kevin Liu, Andrew Cakana, Helgi van de Velde, Jesús F. San Miguel

\section{REFERENCES}

1. Brenner $\mathrm{H}$, Gondos A, Pulte D: Recent major improvement in long-term survival of younger patients with multiple myeloma. Blood 111:2521-2526, 2008

2. Kastritis $E$, Zervas $K$, Symeonidis $A$, et al: Improved survival of patients with multiple myeloma after the introduction of novel agents and the applicability of the International Staging System (ISS): An analysis of the Greek Myeloma Study Group (GMSG). Leukemia 23:1152-1157, 2009

3. Kumar SK, Rajkumar SV, Dispenzieri A, et al: Improved survival in multiple myeloma and the impact of novel therapies. Blood 111:2516-2520, 2008

4. Facon T, Mary JY, Hulin C, et al: Melphalan and prednisone plus thalidomide versus melphalan and prednisone alone or reduced-intensity autologous stem cell transplantation in elderly patients with multiple myeloma (IFM 99-06): A randomised trial. Lancet 370:1209-1218, 2007

5. Mateos MV, Hernandez JM, Hernandez MT, et al: Bortezomib plus melphalan and prednisone in elderly untreated patients with multiple myeloma: Results of a multicenter phase 1/2 study. Blood 108:2165-2172, 2006

6. Palumbo A, Bringhen $S$, Liberati AM, et al: Oral melphalan, prednisone, and thalidomide in elderly patients with multiple myeloma: Updated results of a randomized, controlled trial. Blood 112:3107-3114, 2008

7. San-Miguel J, Harousseau JL, Joshua D, et al: Individualizing treatment of patients with myeloma in the era of novel agents. J Clin Oncol 26:2761-2766, 2008

8. Palumbo A, Rajkumar SV: Treatment of newly diagnosed myeloma. Leukemia 23:449-456, 2009

9. Hulin C, Facon T, Rodon P, et al: Efficacy of melphalan and prednisone plus thalidomide in patients older than 75 years with newly diagnosed multiple myeloma: IFM 01/01 Trial. J Clin Oncol 27:3664-3670, 2009

10. Waage $A$, Gimsing $P$, Juliusson $G$, et al: Melphalan-prednisone-thalidomide to newly diagnosed patients with multiple myeloma: A placebo controlled randomised phase 3 trial. Blood 110:32a, 2007 (suppl; abstr 78)

11. Wijermans $P$, Schaafsma $M$, Van Norden $Y$, et al: Melphalan + prednisone versus melphalan + prednisone + thalidomide in induction therapy for multiple myeloma in elderly patients: Final analysis of the Dutch Cooperative Group HOVON 49 study. Blood 112:241, 2008 (suppl; abstr 649)

12. Barlogie $B$, Tricot $G$, Anaissie $E$, et al: Thalidomide and hematopoietic-cell transplantation for multiple myeloma. N Engl J Med 354:1021-1030, 2006
13. Barlogie $B$, Pineda-Roman $M$, van Rhee $F$, et al: Thalidomide arm of total therapy 2 improves complete remission duration and survival in myeloma patients with metaphase cytogenetic abnormalities. Blood 112:3115-3121, 2008

14. Morgan GJ, Jackson GH, Davies FE, et al: Maintenance thalidomide may improve progression free but not overall survival; results from the Myeloma IX maintenance randomisation. Blood 112: 245, 2008 (suppl; abstr 656)

15. San Miguel JF, Schlag R, Khuageva NK, et al: Bortezomib plus melphalan and prednisone for initial treatment of multiple myeloma. N Engl J Med 359:906-917, 2008

16. Bladé J, Samson D, Reece D, et al: Criteria for evaluating disease response and progression in patients with multiple myeloma treated by high-dose therapy and haemopoietic stem cell transplantation: Myeloma Subcommittee of the EBMT. Br J Haematol 102:1115-1123, 1998

17. Greipp PR, San Miguel J, Durie BG, et al: International staging system for multiple myeloma. $\mathrm{J}$ Clin Oncol 23:3412-3420, 2005

18. Conner TM, Doan QD, Walters IB, et al: An observational, retrospective analysis of retreatment with bortezomib for multiple myeloma. Clin Lymphoma Myeloma 8:140-145, 2008

19. Hrusovsky I, Emmerich B, von Rohr A, et al: Bortezomib retreatment in relapsed multiple myeloma (MM): Results from a binational, multicenter retrospective survey. Blood 112:957, 2008 (suppl; abstr 2775)

20. Petrucci MT, Blau IW, Corradini $P$, et al Efficacy and safety of re-treatment with bortezomib (Velcade()) in patients with multiple myeloma: Results from a prospective international phase II trial. Blood 112:1264, 2008 (suppl; abstr 3690)

21. Rubio-Martinez $A$, Recasens $V$, Soria $B$, et al: Response to re-treatment on relapse multiple myeloma patients previously treated with bortezomib. Haematologica 93:261, 2008 (suppl; abstr 649)

22. Wolf J, Richardson PG, Schuster M, et al: Utility of bortezomib retreatment in relapsed or refractory multiple myeloma patients: A multicenter case series. Clin Adv Hematol Oncol 6:755-760, 2008

23. Mateos MV, Hernandez JM, Hernandez MT, et al: Bortezomib plus melphalan and prednisone in elderly untreated patients with multiple myeloma: Updated time-to-events results and prognostic factors for time to progression. Haematologica 93:560-565, 2008

24. Mateos MV, Oriol A, Martinez J, et al: Bortezomib (Velcade)-melphalan-prednisone (VMP) versus Velcade-thalidomide-prednisone (VTP) in elderly untreated multiple myeloma (MM) patients. Haematologica 94:190, 2009 (suppl; abstr 471)

25. Palumbo $A$, Bringhen $S$, Rossi $D$, et al: Bortezomib, melphalan, prednisone and thalidomide (VMPT) versus bortezomib, melphalan and prednisone (VMP) in elderly newly diagnosed myeloma patients: A prospective, randomized, phase III study. Haematologica 94:190-191, 2009 (suppl; abstr 472)

26. Cavo M, Tacchetti P, Patriarca F, et al: Superior complete response rate and progression-free survival after autologous transplantation with up-front Velcadethalidomide-dexamethasone compared with thalidomidedexamethasone in newly diagnosed multiple myeloma. Blood 112:65, 2008 (suppl; abstr 158)

27. Harousseau JL, Mathiot $C$, Attal $M$, et al: Bortezomib/dexamethasone versus VAD as induction prior to autologous stem cell transplantion (ASCT) in previously untreated multiple myeloma (MM): Updated data from IFM 2005/01 trial. J Clin Oncol 26:455s, 2008 (suppl; abstr 8505)

28. Rosiñol L, Oriol A, Mateos MV, et al: Phase II pethema trial of alternating bortezomib and dexamethasone as induction regimen before autologous stemcell transplantation in younger patients with multiple myeloma: Efficacy and clinical implications of tumor response kinetics. J Clin Oncol 25:4452-4458, 2007

29. Richardson $P$, Lonial $S$, Jakubowiak $A$, et al: Lenalidomide, bortezomib, and dexamethasone in patients with newly diagnosed multiple myeloma: Encouraging efficacy in high risk groups with updated results of a phase I/II study. Blood 112:41, 2008 (suppl; abstr 92)

30. Palumbo A, Schlag $R$, Khuageva $N$, et al: Prolonged therapy with bortezomib plus melphalanprednisone (VMP) results in improved quality and duration of response in the phase III VISTA study in previously untreated multiple myeloma (MM). Haematologica 93:83, 2008 (suppl; abstr 207)

31. Richardson PG, Xie W, Mitsiades $C$, et al: Single-agent bortezomib in previously untreated multiple myeloma: Efficacy, characterization of peripheral neuropathy, and molecular correlates of response and neuropathy. J Clin Oncol 27:35183525, 2009

32. Richardson PG, Sonneveld P, Schuster MW, et al: Reversibility of symptomatic peripheral neuropathy with bortezomib in the phase III APEX trial in relapsed multiple myeloma: Impact of a dose-modification guideline. $\mathrm{Br} J$ Haematol 144:895-903, 2009 